\title{
Mapping and identification of CsUp, a gene encoding an Auxilin-like protein, as a putative candidate gene for the upward- pedicel mutation (up) in cucumber
}

Jingxian Sun, Tingting Xiao, Jingtao Nie, Yue Chen, Duo Lv, Ming Pan, Qifan Gao, Chunli Guo, Leyu Zhang, Huan-Le He, Hongli Lian, Junsong Pan, Run Cai and Gang Wang ${ }^{*}$ (D)

\begin{abstract}
Background: Pedicel orientation can affect the female flower orientation and seed yield in cucumber. A spontaneous mutant possessing upward growth of pedicels was identified in the wild type inbred strain 9930 and named upward-pedicel (up). The morphological and genetic analyses of up were performed in this study. In order to clone the up gene, $933 \mathrm{~F}_{2}$ individuals and $524 \mathrm{BC}_{1}$ individuals derived from C-8-6 (WT) and up were used for map-based cloning.

Results: up was mapped to a $35.2 \mathrm{~kb}$ physical interval on chromosome 1, which contains three predicted genes. Sequencing analysis revealed that a 5-bp deletion was found in the second exon of Csa1G535800, and it led to a frameshift mutation resulting in a premature stop codon. The candidate gene of CsUp (Csa1G535800) was further confirmed via genomic and cDNA sequencing in biparental and natural cucumber populations. Sequencing data showed that a 4-bp deletion was found in the sixth exon of Csa1G535800 in CGN19839, another inbred line, and there was also a mutation of an amino acid in Csa1G535800 that could contribute to the upward growth of pedicels in CGN19839. Moreover, it was found that Csa1G535800 exhibited strong expression in the pedicel of WT, suggesting its important role in development of pedicel orientation. Thus, Csa1G535800 was considered to be the candidate gene of Csup.

Conclusions: CsUp encodes an Auxilin-like protein and controls pedicel orientation in cucumber. The identification of CsUp may help us to understand the mechanism of pedicel orientation development and allow for investigation of novel functions of Auxilin-like proteins in cucumber.
\end{abstract}

Keywords: Cucumis sativus, Upward-pedicel, Map-based cloning, Pedicel orientation, Auxilin-like protein

\section{Background}

Cucumber (Cucumis sativus $\mathrm{L} ., 2 \mathrm{n}=2 \mathrm{x}=14$ ) is an economically important vegetable crop cultivated worldwide. It was first domesticated in India and is currently widely grown in China [1-4]. In cucumber, most organic nutrients of stalk and fruit was transported in via the pedicel [5]. The orientation of the pedicel, a critical organ in various plants, is also an important plant architectural trait that can influence flower position and

\footnotetext{
* Correspondence: wg770801@sjtu.edu.cn

School of Agriculture and Biology, Shanghai Jiao Tong University, 800

Dongchuan Road, Minhang District, Shanghai 201100, China
}

orientation and thus affect pollination, pollinator attraction, insect foraging behaviour, pollen transfer and seed production [6-14].

In recent years, pedicel orientation has been studied extensively. In Arabidopsis, CRM1/BIG, an auxin transport-related gene, was found to encode a membrane-associated protein affecting pedicel orientation [15-20]. In the crm1/big mutant, the pedicels and internodes were very short and downward-bending $[15,19]$. In addition to CRM1/BIG, several genes in Arabidopsis were also reported to be involved in regulating pedicel orientation, including BP, KNAT2, KNAT6, AS1, AS2, ATH1 and

(c) The Author(s). 2019 Open Access This article is distributed under the terms of the Creative Commons Attribution 4.0 International License (http://creativecommons.org/licenses/by/4.0/), which permits unrestricted use, distribution, and 
$L E A F Y$ [16, 17, 20-27]. In tobacco, NtBPL, the homologous gene of $B P$ in Arabidopsis, was found to be involved in pedicel elongation but its effect on pedicel orientation is limited [16, 28]. In tomato, overexpression of SlAGO7 could lead to a dramatic change in pedicel morphology and cause the pedicels to be upward-pointing [29]. In pepper, Capana12g000943 was predicted to be an important player in pedicel orientation development by transporting cytokinin across the plasma membrane [30]. However, in cucumber, most studies were mainly focused on flower sex determination, fruit skin colour, compact plant architecture, powdery mildew resistance, and trichomes formation. The mechanism of pedicel orientation development remains elusive.

Most cucumber inbred lines possess downward growth of pedicels with downward-facing or horizontal-facing female flowers. In the present study, a spontaneous upward-pedicel mutant named upward-pedicel (up) was derived from wild type 9930. The pedicel of $u p$ always displays upward growth with an upward-facing flower until 5 days after pollination (DAP). After 5 DAP, the pedicel turns downward in response to the increasing fruit weight. In this study, we report the map-based cloning of $u p$ and demonstrate that it encodes an Auxilin-like protein that might play an important role in pedicel orientation development in cucumber. This finding can help us to understand the mechanism of pedicel orientation development in cucumber.

\section{Results}

The up phenotype was controlled by a recessive nuclear gene in cucumber

In WT, the pedicel orientation remained horizontal when the young fruits were at the early stage. After that, their pedicel became downward-bending and the orientation of the pedicel remained downward-pointing (Fig. 1a, b, c, d and e). In up, the pedicel showed upward growth before 3 days after pollination and then the pedicel became downward-bending with the increasing fruit weight (Fig. 1f, g, h, i and j).

The pedicel orientation of the $F_{1}$ plants produced from the cross of $u p$ and WT was the same as WT. No significant phenotypic difference was found between homozygous dominant ( $U p / U p$ ) and heterozygous (Up/up) plants. In a small $F_{2}$ population with 133 individuals, the downward-pedicel plants and upward-pedicel plants fitted a segregation ratio of $3: 1$ (100:33). In a large $\mathrm{BC}_{1}$ population with 524 individuals, the downward-pedicel plants and upward-pedicel plants fitted a segregation ratio of 1:1 (255:269). In a large $F_{2}$ population with 800 individuals, there were 599 downward-pedicel individuals and 201 upward-pedicel individuals, which also fitted the 3:1 segregation ratio (Table.1). These results suggested that both the $\mathrm{F}_{2}$ population and the $\mathrm{BC}_{1}$ population conformed to Mendel segregation which confirmed that $u p$ was conferred by a single recessive locus in cucumber.

\section{Primary mapping}

Based on the re-sequencing genomic data, 95 polymorphic InDel markers between $u p$ and WT were developed. All of the 41 polymorphic SSR markers and 95 polymorphic InDel markers between $u p$ and WT were screened to analyse the WT and M DNA pools using a BSA strategy. Among these markers, seven markers on cucumber chromosome 1 showed polymorphism between the WT and M DNA pool (Additional file 1: Figure S1). The seven markers were used for linkage analysis by using $70 \mathrm{~F}_{2}$ individuals which were randomly selected from the small $F_{2}$ population. The results showed that the up locus was located on chromosome 1, flanked by the closer markers s-indel1-6 and s-indel1-23, at a genetic distance of 2.4 and $5.3 \mathrm{cM}$, respectively (Fig. 2a). Based on the genomic sequence, three new polymorphic InDel markers (S-indel1-66, S-indel1-75 and S-indel1-70) between s-indel1-6 and s-indel1-23 were developed and the three markers were used for further mapping. The up locus was flanked by closer markers s-indel1-75 and s-indel1-70. These two markers (s-indel1-75 and s-indel1-70) were used to screen the $\mathrm{F}_{2}$ population (small $\mathrm{F}_{2}$ population and large $\mathrm{F}_{2}$ population) and the large $\mathrm{BC}_{1}$ population. $\mathrm{Fi}$ nally, two markers s-indel1-75 and s-indel1-70 showed 13 and 26 recombinants among $933 \mathrm{~F}_{2}$ individuals and $524 \mathrm{BC}_{1}$ individuals, respectively (Fig. 2b).

\section{Fine mapping of the up locus}

The physical distance between s-indel1-75 and s-indel1-70 was approximately $785.1 \mathrm{~kb}$ according the '9930' genome database (http://cucurbitgenomics.org/organism/2). The resequencing genome data showed that there were no more polymorphic InDel markers between s-indel1-75 and s-indel1-70. Therefore, SNP markers between s-indel1-75 and s-indel1-70 were developed for fine mapping. Eleven SNP markers were developed and these SNP markers showed polymorphisms in the parents. Out of $933 \mathrm{~F}_{2}$ individuals and $524 \mathrm{BC}_{1}$ individuals, 39 recombinants between s-indel1-75 and s-indel1-70 were used for fine mapping with the polymorphic SNP markers. Finally, the up locus was narrowed to a region between S-SNP1-35 and S-SNP1-40 markers, encompassing a physical distance of $35.2 \mathrm{~kb}$ (Fig. 2c) and each of two markers with one and two recombinants, respectively. The marker S-SNP1-37 was co-segregated with the up locus between S-SNP1-35 and S-SNP1-40. 

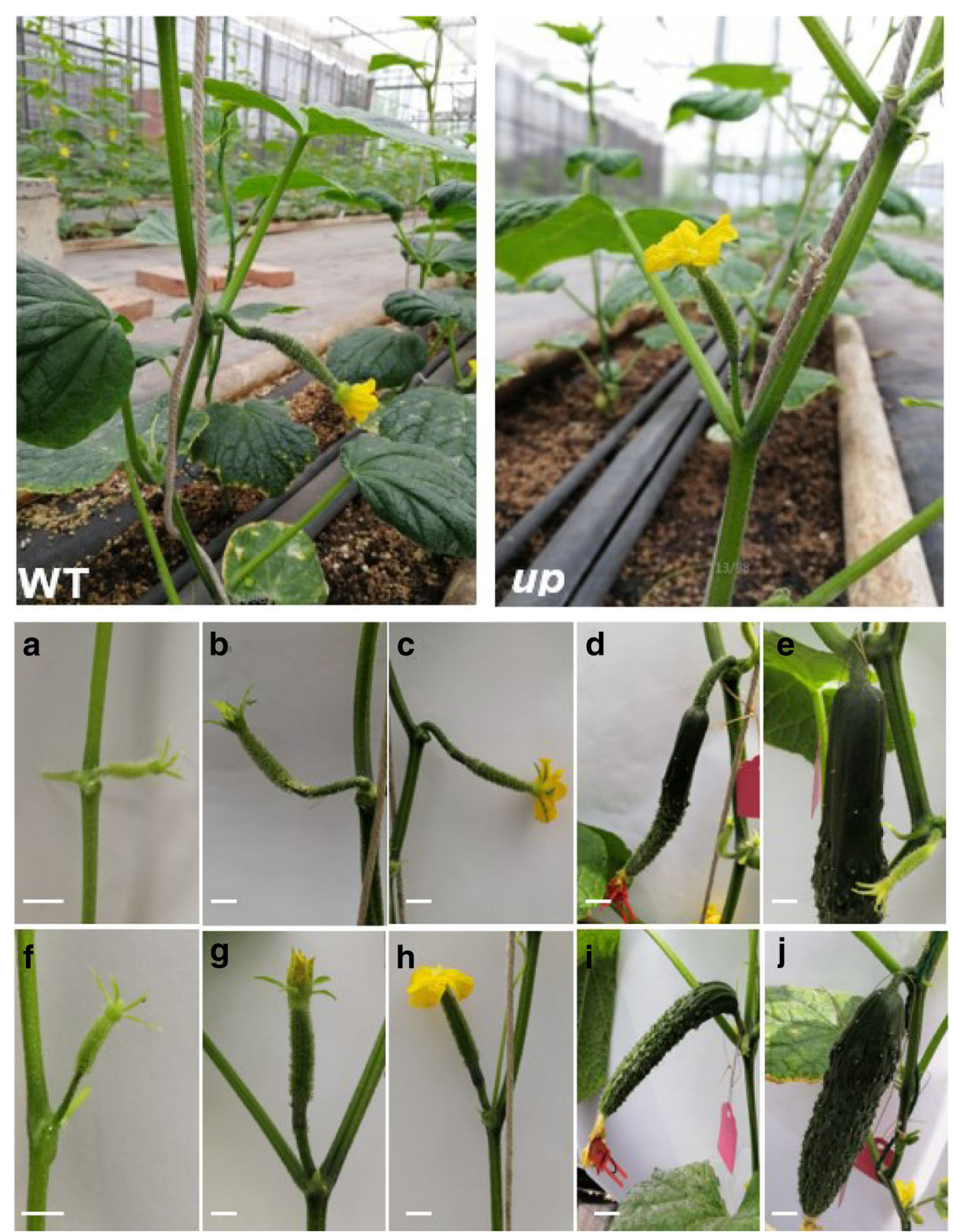

Fig. 1 Phenotypic features of the pedicel in WT and up, and the pedicel orientation at different stages in two parents. $\mathbf{a}, \mathbf{b}, \mathbf{c}, \mathbf{d}$ and $\mathbf{e}$ are pedicels from WT. $\mathbf{f}, \mathbf{g}, \mathbf{h}, \mathbf{i}$ and $\mathbf{j}$ are pedicels from up. $\mathbf{a}, \mathbf{f}$ Young fruits at an early stage (approximately $1 \mathrm{~cm}$ ); $\mathbf{b}, \mathbf{g}$ Young fruits of the day before female flowers open; $\mathbf{c}, \mathbf{h}$ Young fruits with opening female flowers; $\mathbf{d}$, $\mathbf{i}$ Fruits 3 days after pollination; $\mathbf{e}, \mathbf{j}$ Fruits 5 days after pollination. When the female flowers were blooming or bloomed, the pedicel was downward growth in WT and upward growth in up

Table 1 Segregation analysis of the up trait in the $F_{2}$ population (WT $\times$ up)

\begin{tabular}{llllllll}
\hline Population & \multicolumn{3}{l}{ Number of plants } & Expected ratio & $x^{2 a}$ & $P$ \\
\cline { 2 - 4 } & 20 & 20 & 0 & & & & \\
\hline$F_{1}$ & 133 & 100 & 33 & $3: 1$ & - & - \\
$F_{2}$ & 800 & 607 & 193 & $3: 1$ & 0.003 & 0.96 \\
$F_{2}$ & 524 & 255 & 269 & $1: 1$ & 0.003 & 0.33 \\
$\mathrm{BC}_{1}$ & & & & & & \\
\hline
\end{tabular}

$D$ : downward-pedicel; U: upward-pedicel a $\times 2(0.05,1)=3.84$

\section{Predicted genes in the up region}

In the $35.2 \mathrm{~kb}$ DNA segment, three putative genes were identified in the '9930' genome database (http://cucurbitgenomics.org/organism/2). The detailed information about these genes is summarized in Fig. 3a and Table 2. DNA sequence of the three genes and their promoters (2000 bp before start codon) was amplified and analysed. Compared to WT, there was no polymorphism in both promoter sequence and gene sequence of Csa1G535810 (Additional file 10: Figure S9, Additional file 13: Figure S12). In Csa1G535790, there was only one SNP 


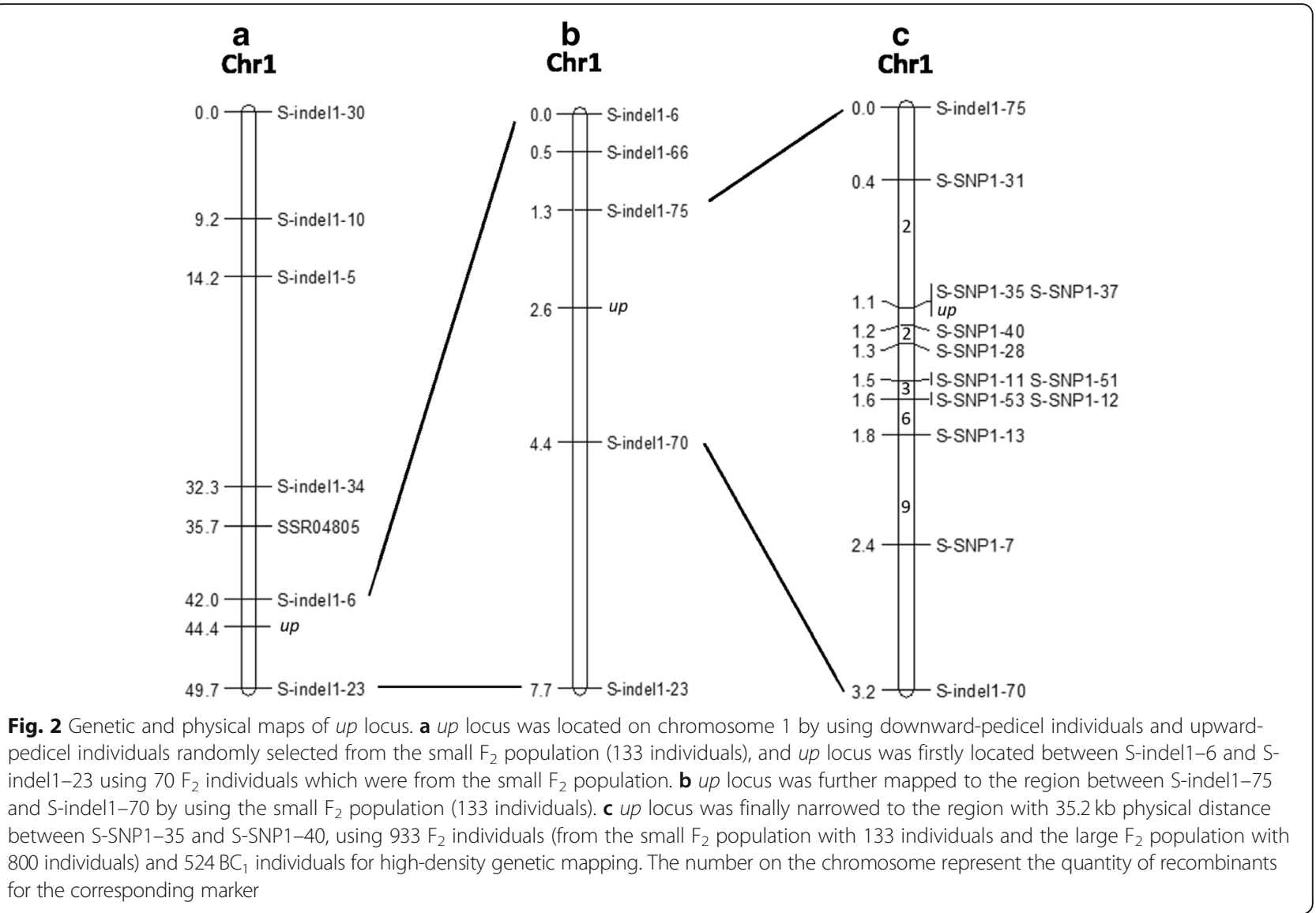

polymorphism in the promoter region at $-611 \mathrm{bp}$ position (Additional file 12: Figure S11) and no polymorphism in the gene sequence (Additional file 9: Figure S8). In Csa1G535800, there was a 5-bp deletion in the second exon (Additional file 7: Figure S6) and no polymorphism in the promoter sequence (Additional file 11: Figure S10). cDNA sequence amplification and analysis revealed an identical 5-bp deletion at the same locus in up (Additional file 8: Figure S7). Further analysis revealed that the 5 -bp deletion caused a frameshift mutation and resulted in a premature stop codon at 612-614 bp in the mRNA (Fig. 3c). Protein BLAST searches indicated that the gene Csa1G535800 was predicted to encode an Auxilin-like protein with a conserved DnaJ-domain at the C-terminal (Fig. 4). Due to the 5-bp deletion, a large segment of amino acid sequence including the DnaJ domain was lost in the predicted protein (Fig. 4). Although there is a SNP in the promoter of Csa1G535790, we think that Csa1G535800 was the most likely candidate gene.

\section{Identification of the putative candidate gene for up}

To further confirm Csa1G535800 was the candidate gene, we examined linkage relationship between the 5-bp deletion and the up locus. An InDel marker
(Indel-CsUp) developed from the fragment with the 5 -bp deletion was used for genotyping the $\mathrm{F}_{2}$ population with 800 individuals. Genotype analysis showed that the marker Indel-CsUp co-segregated with the up locus in this $F_{2}$ population (Additional file 2: Figure S2). Furthermore, we analysed the coding sequences of 19 cucumber inbred lines based on their resequencing data (Additional file 8: Figure S7). Among the 19 cucumber inbred lines, there was a pair of near-isogenic lines B1 (upward-pedicel) and B2 (downward-pedicel), and all of the inbred lines had downward-pedicels except B1 and CGN19839 (Additional file 3: Figure S3). Sequence analysis showed that all of the inbred lines with downward growth pedicels shared the same sequence with WT in the encoding region. Moreover, in B1, we found the same 5-bp deletion in the encoding region. In CGN19839, we found an A/G transition in exon 1 that caused an amino acid change from Ile (I) to Val (V) and a 4-bp deletion in exon 6 that led to a premature stop codon at 1243-1245 bp in the mRNA. The 4-bp deletion could also result in the DnaJ domain being lost from the predicted protein. To confirm the result, genomic DNA and cDNA of Csa1G535800 from B1 and CGN19839 were sequenced and the results were consistent with the resequencing data. 


\section{S-SNP 1-35 \\ $35.2 \mathrm{~kb}$ \\ S-SNP 1-40}

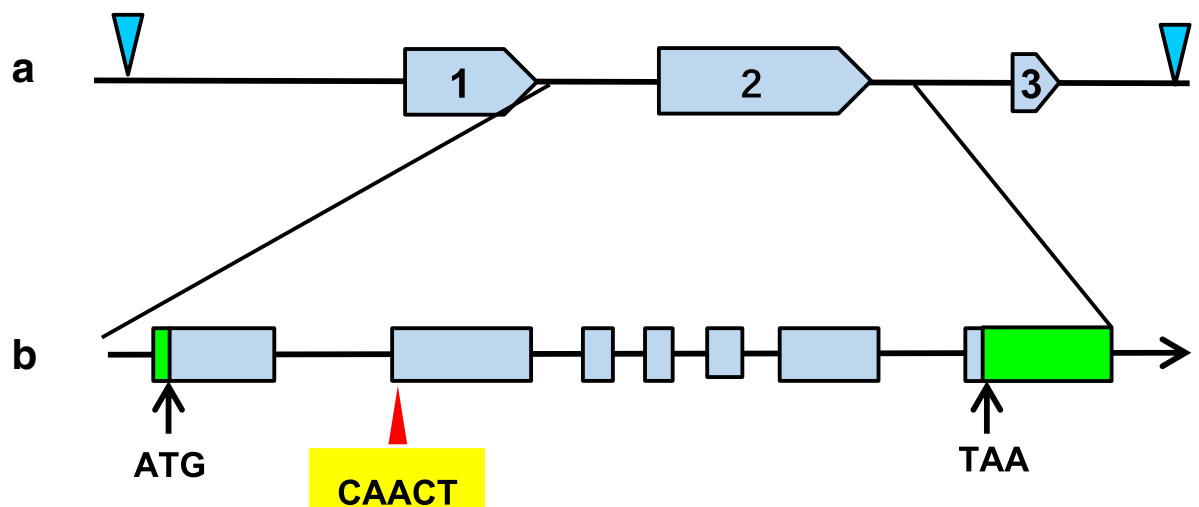

5-bp deletion in up

$\begin{array}{ll}\text { WT } & \text { AATTCATACACAACTATGTTCAAGGAACAGGAAATGCCACAGTTTGCACCTCATCTCTCC } 540 \\ 9930 & \text { AATTCATACACAACTATGTTCAAGGAACAGGAAATGCCACAGTTTGCACCTCATCTCTCC } 540 \\ \text { c } & \text { AATTCATACA----ATGTTCAAGGAACAGGAAATGCCACAGTTTGCACCTCATCTCTCC } 535 \\ & \\ \text { WT } & \text { CCTCATATGGATAACCGTTATGTAGAAGATGAATATGATGATAGATACAAAAGCTCAGAC } 600 \\ 9930 & \text { CCTCATATGGATAACCGTTATGTAGAAGATGATATGATGATAGATACAAAAGCTCAGAC } 600 \\ u p & \text { CCTCATATGGATAACCGTTATGTAGAAGATGAATATGATGATAGATACAAAAGCTCAGAC } 595\end{array}$

Fig. 3 Representation of the three putative genes between S-indel1-75 and S-indel1-70. a There were three putative genes (blue boxes) between S-SNP1-35 and S-SNP1-40. Gene numbers correspond to those in Table 2. b Structure of Csa1G535800. Boxes and lines indicate exons and introns, respectively. Two green boxes in Csa1G535800 indicate the 5' UTR and the 3' UTR. Sequencing results revealed that, compare to WT, up has a 5-bp deletion at 882-886 bp from the ATG start codon in the second exon of Csa1G535800. The yellow box shows the deletion sequence in up. c The position of the 5-bp deletion in the coding sequence. Sequencing results revealed that there was a 5-bp deletion at 491-495 bp from the ATG start codon that resulted in a premature stop codon at 612-614 bp in the mRNA

\section{Phylogenetic relationships of CsUp with its homologs in other species}

Protein BLAST searches indicated that the gene Csa1G535800 encodes an Auxilin-like protein (CsUp) with a conserved DnaJ-domain, and this DnaJ-domain contains 44 amino acid residues (Fig. 4). To better understand the genetic and functional relationships of CsUp between cucumber and other species, a phylogenetic tree was constructed on the basis of full-length protein sequences of $\mathrm{CsUp}$ and 11 other homologous proteins (Fig. 5). Amino acid sequence identity between $\mathrm{CsUp}$ and other homologs varied from 40 to $95 \%$. In the phylogenetic tree, the CsUp was more similar to LOC103487941 (Cucumis melo) and
At1G30280 (Arabidopsis thaliana). In Cucumis melo, LOC103487941 is an uncharacterized protein and its function is unclear. In Arabidopsis, At1G30280 is a chaperone DnaJ-domain superfamily protein named Auxilin-like7, however, limited work has been performed on the functional characterization of this gene.

\section{Transcript expression analysis of Csa1G535800}

Pattern expression of Csa1G535800 was examined in roots, stems, true leaves, female flowers, male flowers, fruits and pedicels from WT and $u p$ by qRT-PCR (Fig. 6). Csa1G535800 was mainly expressed in the pedicel and female flower. The expression level showed no significant differences between WT and up in roots, stems,

Table 2 Predicted genes between markers S-SNP1-35 and S-SNP1-40

\begin{tabular}{|c|c|c|c|c|c|}
\hline \multirow{2}{*}{$\begin{array}{l}\text { Gene } \\
\text { No. }\end{array}$} & \multicolumn{2}{|c|}{ Predicted genes (ID) } & \multirow[t]{2}{*}{ Gene function } & \multirow{2}{*}{$\begin{array}{l}\text { BLASTX } \\
\text { plant } \\
\text { proteins }\end{array}$} & \multirow{2}{*}{$\begin{array}{l}E- \\
\text { value }\end{array}$} \\
\hline & $\mathrm{Cmb}$ & Phytozome v10 & & & \\
\hline 1 & Csa1G535790 & Cucsa.152130 & Putative mitochondrial transcription termination factor family protein & AT2G34620 & $1 e-128$ \\
\hline 2 & Csa1G535800 & Cucsa.152140.1 & Auxilin-like protein & AT1G30280 & $4 e-10$ \\
\hline 3 & Csa1G535810 & Not found & Unnamed protein & Not found & - \\
\hline
\end{tabular}




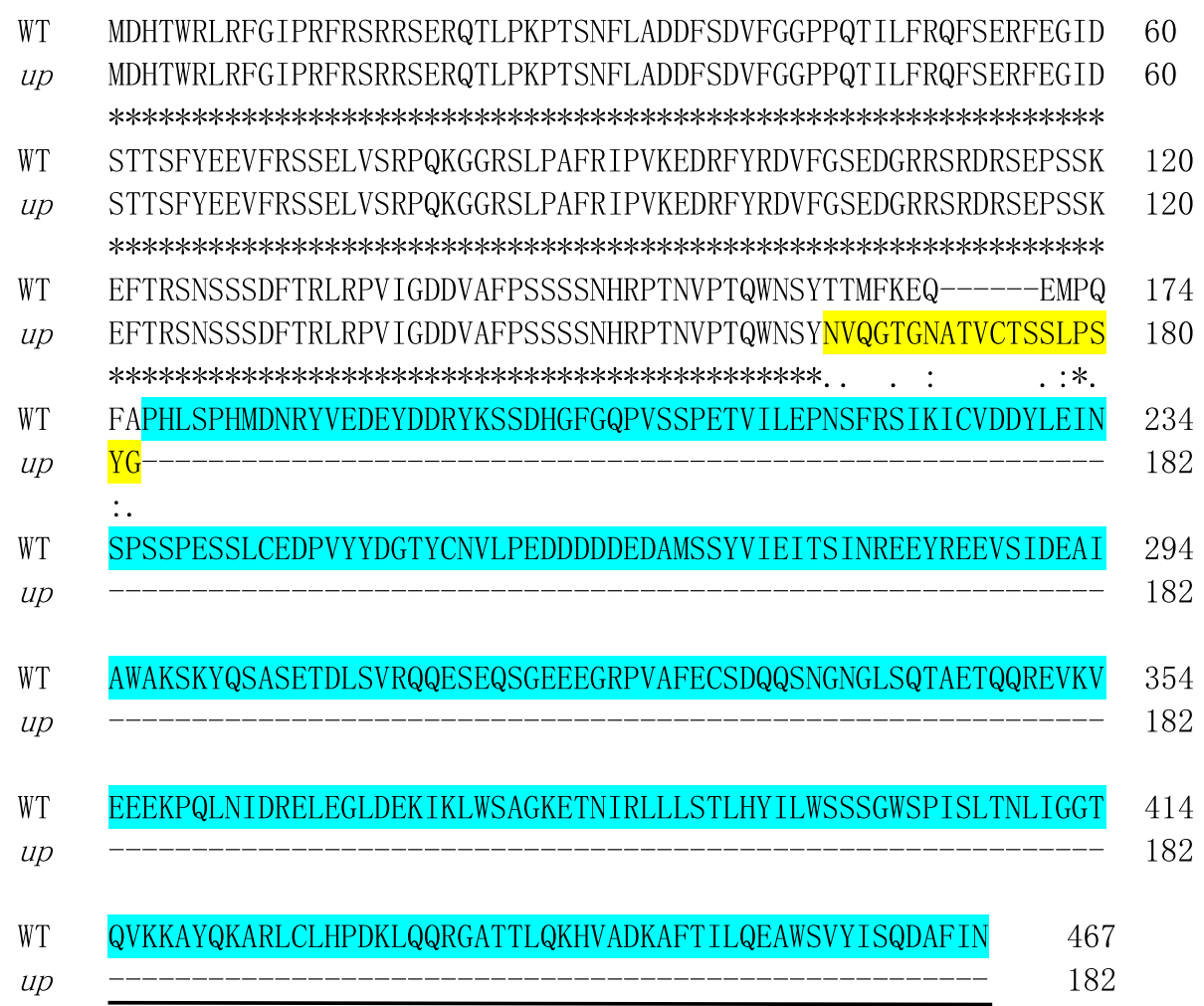

Fig. 4 Alignment of predicted protein sequences of Csa1G535800 between WT and up. The 5-bp deletion site resulted in the introduction of a premature stop codon, and the encoded protein lacked the normal amino acids from position 164. The amino acids highlighted in yellow are abnormal in up and the amino acids highlighted in blue are missing in up. The DnaJ domain at the C-terminal is underlined

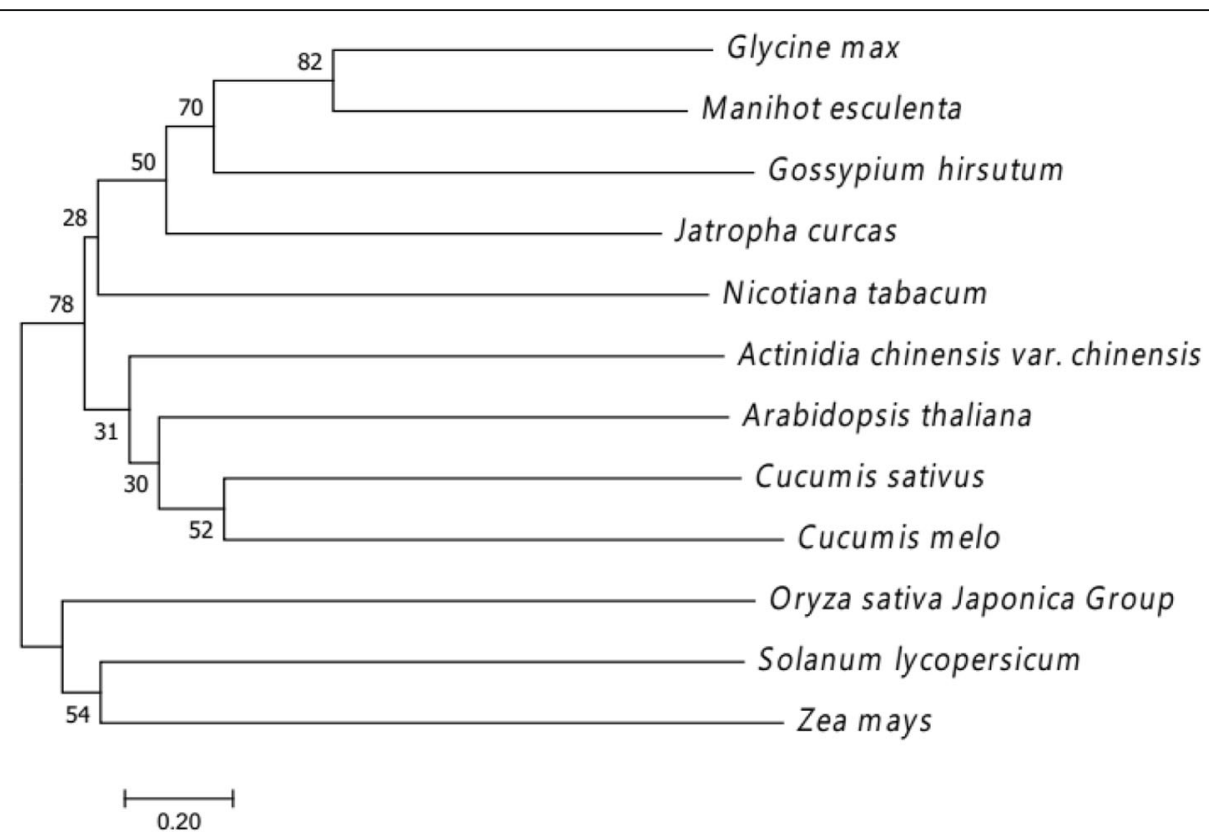

Fig. 5 Phylogenetic tree of CsUP in cucumber and its homologs in other species. The phylogenetic tree was constructed using the neighbourjoining method built in MEGA 7, and the percentage of replicate trees in which the associated taxa clustered together in the bootstrap test (1000 replicates) are shown next to the branches. Numbers on the tree represented bootstrap values 


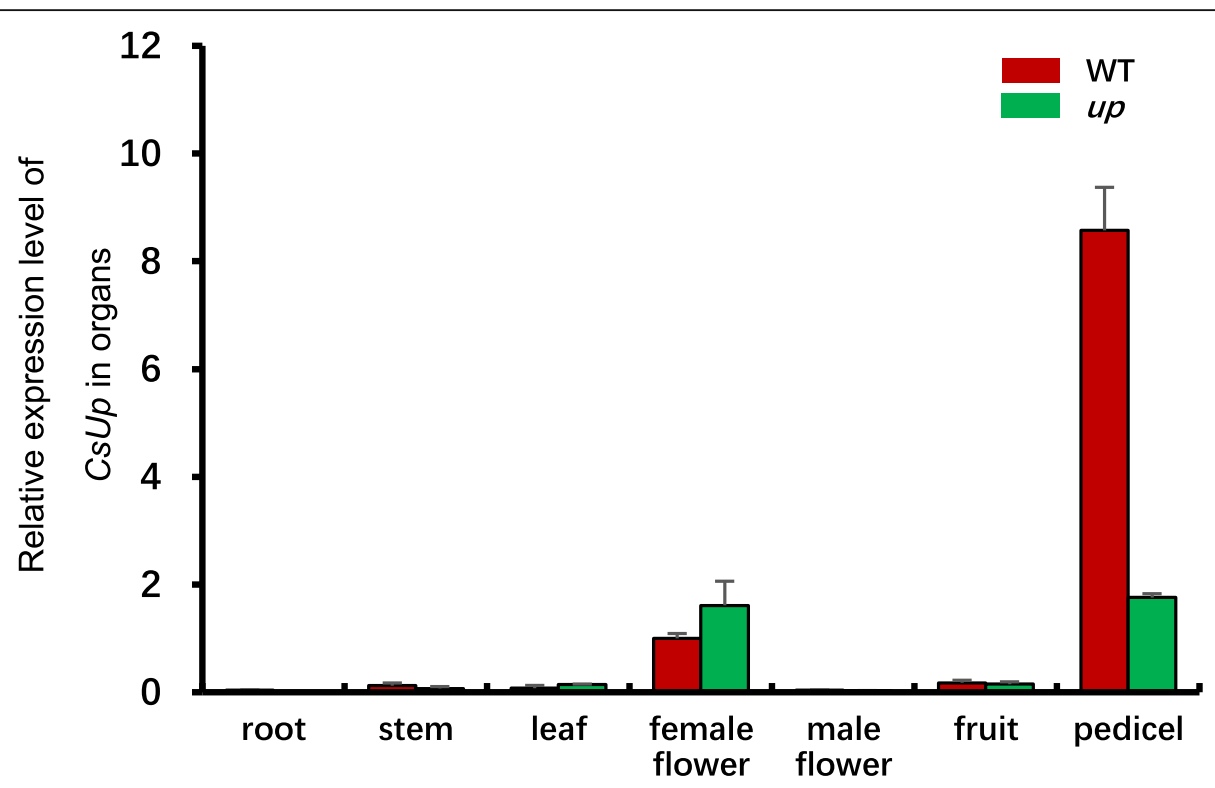

Fig. 6 Expression level of CsUp candidate gene in seven organs of WT and up. Analysis of relative transcript abundance used female flowers in WT as the reference. Data are displayed as the ratio of expression to CsActin3 with three biological replicates. Error bars represent standard error (SE)

true leaves, female flowers, male flowers or fruits. In up, the expression level in the pedicel was significantly lower than that in WT. The expression level in WT was approximately 6.5-fold higher than that in up. We examined the temporal expression of Csa1G535800 in the pedicels that were derived from 1 to $1.5 \mathrm{~cm}$ length fruits, the young fruits of the day before female flowers open and the young fruits with opening female flowers (Fig. 7). The expression results showed that, within WT and $u p$, there was no significant difference temporally among the three stages. However, compared to WT, the expression level was significantly lower in $u p$ at the three stages, which is consistent with the results of pattern expression.

The temporal expression of Csa1G535790 and Csa1G535810 was also examined in the pedicels that were derived from 1 to $1.5 \mathrm{~cm}$ length fruits, the young fruits of the day before the female flowers open and the young fruits with opening female flowers (Additional file 14: Figure S13). Within WT and up, the transcription level of Csa1G535790 and Csa1G535810 was no clear difference among the three stages, and there was also no

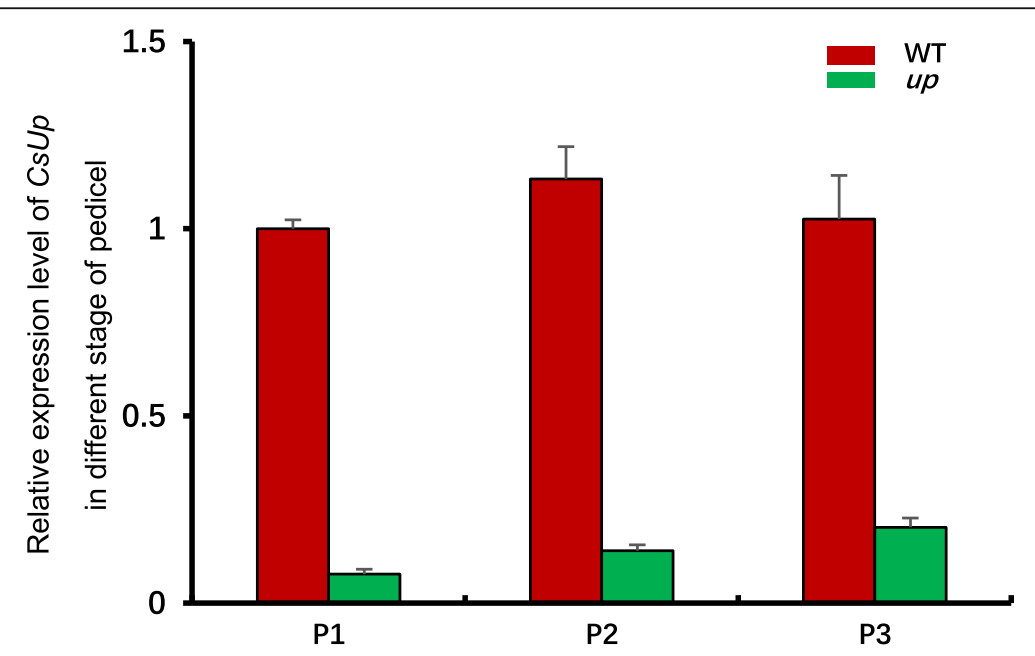

Fig. 7 Expression level of CsUp candidate gene in different stage of pedicel. P1 pedicels from 1 to $1.5 \mathrm{~cm}$ length young fruits; P2 pedicels from the young fruits the day before the female flowers open; P3 pedicels from the young fruits with opening female flowers. Data are displayed as the ratio of expression to CsActin3 with three biological replicates. Error bars represent standard error (SE) 
significant difference between WT and $u p$. Therefore, the SNP polymorphism from the promoter region of Csa1G535790 may not influence the gene expression level.

\section{Discussion}

In this study, we isolated the candidate gene (CsUp) for upward-pedicels based on map cloning using a spontaneous upward-pedicel mutant ' $u p$ '. Sequencing results revealed that a 5-bp deletion was found in the second exon of $C s U p$, and this deletion resulted in loss of a large segment of amino acids in the predicted protein (Fig. 3). After analysing the $F_{2}$ population with 800 individuals, we found that the marker indel-CsUp was co-segregated with the upward-pedicel phenotype (Additional file 2: Figure S2). In B1 (upward-pedicel), the same 5-bp deletion was found in CsUp, but this deletion did not exist in its near-isogenic line B2 (downward-pedicel). Furthermore, a different mutation type was found in another up inbred line CGN19839. Sequencing data showed that one A/G transition at exon 1 caused an amino acid change from Ile (I) to Val (V) and a 4-bp deletion in the exon 6 of CsUp led to a premature stop codon (Additional file 4: Figure S4). We speculated that the two kinds of mutations can cause dysfunction of CsUp and result in the upward-pedicel phenotype in up, B1 and CGN19839. In WT, the expression level of CsUp was the highest in the pedicel, which supported $C s U p$ being the most likely candidate gene of $u p$ (Figs. 6 and 7).

Although there was a SNP polymorphism in the promoter region of Csa1G535790 (Additional file 12: Figure S11), while there was no clear transcription difference between WT and $u p$ among the three stages (Additional file 14: Figure S13). Therefore, we think the SNP polymorphism in the promoter region of Csa1G535790 may not the reason of $u p$ mutant phenotype.

Pedicel is an important plant architecture trait and its orientation affects flower orientation. In other plants, flower orientation is an important characteristic that influences reproductive success and yield. In the plants with complex inflorescences, the flower orientation affects pollinator behaviour and pollination efficiency [14]. In single flower plants, the flower orientation often plays an important role in protecting flowers from bad environments $[8,9]$. As single flower plants, most cucumber inbred lines present their female flowers horizontally or downward-facing. Under natural growth conditions, female flowers may employ the same protective strategy as found in other single flower plants. In $u p$, when the upward-facing female flowers are blooming in natural growth conditions, the pistil stigma will lose the protection of the petals and may be vulnerable to rain drops or direct sunlight. The reproduction of cucumbers may be influenced by bad weather. We speculated that the horizontal or downward-facing female flowers in cucumber are probably due to natural selection.

To date, a number of underlying molecular regulators controlling the pedicel orientation have been uncovered, including Arabidopsis LEAFY [27], KNAT1/BP [16, 21], KNAT2 and KNAT6 [20], CRM1/BIG [19], AS1 [17], AS2 [31] and ATH1 [23]; tomato SlAGO7 [29]; Gloxinia TCP [32]; and tobacco NtSVP [28]. However, none of their homologous proteins was identified in cucumber. In this study, we isolated the gene CsUp that might regulate the development of pedicel orientation directly or indirectly in cucumber. Combining the previous study on pedicel orientation and our present result, we speculated that CsUp maybe a new type of gene regulating the pedicel orientation in plants.

The CsUp, an Auxilin-like protein, contains a conserved DnaJ domain. Bioinformatics analysis showed that the homologous protein of CsUp, AUXILIN-LIKE7, is also an Auxilin-like protein in Arabidopsis. However, the function of AUXILIN-LIKE7 protein is unknown in Arabidopsis. In cucumber, the function of six Auxilin-like proteins has not been reported. In Arabidopsis, there are seven Auxilin-like proteins, named AUXILIN-LIKE1, AUXILIN-LIKE2, AUXILIN-LIKE3, AUXILIN-LIKE4, AUXILIN-LIKE6, AUXILIN-LIKE7 and auxin-like 1. Both AUXILIN-LIKE1 and AUXILIN-LIKE2 are clathrin uncoating factors involved in clathrin-mediated endocytosis (CME) and overexpression of these factors caused an arrest of growth and development and an inhibition of endocytosis [33]. AUXILIN-LIKE6 involved in phototropin-mediated chloroplast movement in Arabisopsis [34]. In rice, $X B 21$, an Auxilin-like protein, shows high homology to AUXILIN-LIKE1 and AUXILIN-LIKE2, and plays an important role in plant immune response and cell death regulation [35]. Thus, we speculated that different Auxilin-like proteins have different functions in the same plant and the homologous genes in different plants might play different roles in plant growth. Therefore, we hypothesized that the six Auxilin-like proteins in cucumber may perform different functions in cucumber growth.

A detailed sequence alignment showed that CsUp harbours a conserved domain, named as DnaJ domin (Fig. 4). DnaJ domain plays critical roles in Auxilin-like proteins $[36,37]$. Auxilin-like protein is a kind of DnaJ protein and plays an important role in clathrin-mediated endocytosis [38, 39]. In $u p$, the 5-bp deletion resulted in the DnaJ domain being lost in the predicted proteins (Fig. 4). In CGN19839, the DnaJ domain was also lost in the predicted proteins caused by the 4-bp deletion (Additional file 5: Figure S5). Furthermore, the A/G transition at exon 1 is far away from the DnaJ domain, thus, this SNP mutation might not inactivate CsUp [37]. These results indicated that the function of $\mathrm{CsUp}$ might be destroyed due to the loss of DnaJ domain. The loss-of-function of CsUp caused by DnaJ domain loss 
might result in the upward-pedicel phenotype in $u p$ and CGN19839.

We examined the pattern expression of CsUp through qRT-PCR (Fig. 6). The results suggested that CsUp was strongly expressed in the pedicel of WT and the expression level was much higher than that in $u p$. We speculated that the loss-of-function of CsUp would no longer work and formed a feedback inhibition in up, which might cause the expression level of CsUp much lower than that in WT. In up, the pedicels always showed upward growth from the initiation of young fruits to female flowers 5 days after pollination (Fig. 1), and we concluded that the lack of the DnaJ domain might destroy the function of CsUp, and thus the expression of CsUp was invariable at different stages in $u p$. The results of temporal expression also confirmed our hypothesis (Fig. 7). These results suggested that the CsUp might play an important role in pedicel orientation development in cucumber. However, how CsUp affects the pedicel orientation in cucumber is unclear. Therefore, additional studies could provide glimpses into the regulatory mechanism of pedicel orientation in cucumber.

\section{Conclusions}

The pedicel orientation in $u p$ mutant is upward growth. $u p$ is controlled by a single recessive gene. The candidate gene CsUp encodes an Auxilin-like protein with the full length of $2613 \mathrm{bp}$. The mutation, 5-bp deletion in the second exon, of CsUp in up resulted in a frameshift mutation and earlier translation termination. The identification of CsUp may help us to understand the mechanism of pedicel orientation and provide investigation of novel functions of Auxilin-like protein in cucumber.

\section{Methods}

\section{Plant materials}

up, a spontaneous mutant possessing upward growth pedicels, was obtained from the wild type 9930. The C-8-6 (WT) is a North China fresh market type inbred line with downward growth pedicels. When the female flowers bloomed, the pedicle of $u p$ exhibited upward growth with upturned female flowers, while in WT, the pedicel exhibited downward growth with horizontal-facing or downward-facing female flowers (Fig. 1). $F_{1}$ plants were derived from the cross between WT and $u p$, then the $\mathrm{F}_{1}$ population was self-pollinated to produce the $F_{2}$ population. The $F_{1}$ population was backcrossed with the recessive parent (up) to obtain the $\mathrm{BC}_{1}$ population. All of the plants were grown under natural sunlight in a greenhouse at Shanghai Jiao Tong University, Shanghai, China.

\section{InDel and SNP markers development based on genome} resequencing

Due to the close genetic relationship and the low polymorphism of SSR markers between up and WT (2\%), deletion-insertion(InDel)and single nucleotide polymorphism(SNP)markers were developed based on the genome resequencing. The genome of WT was sequenced on the Illumina HiSeq 2000 platform (Biomarker Technologies, Beijing, China). With 30 -fold sequencing depth, all of the clean reads were mapped to the "9930" genome sequence (http://cucurbitgenomics.org/organism/2, version 2i). InDel and SNP sites between WT and 9930 were detected with the Genious software package. Only fragments with a more than $3 \mathrm{bp}$ insertion or deletion were used to develop InDel markers. For SNP genotyping, approximately $800 \mathrm{bp}$ fragments including the SNP site in the middle of the fragment were amplified and sequenced. The primers were designed with Primer Premier 5.0.

\section{Phenotypic data collection}

The pedicel orientation data of WT, up, $\mathrm{F}_{1}$ and all individuals from the $\mathrm{F}_{2}$ and $\mathrm{BC}_{1}$ populations were collected when the female flowers were blooming. The data about pedicel orientation were collected from three to five opening female flowers in each plant.

\section{DNA extraction and molecular marker analysis}

Genomic DNA was extracted from young leaves using the CTAB method [40]. For the SSR and InDel markers, PCR reactions were carried out using a $10 \mu \mathrm{l}$ volume containing $40 \mathrm{ng}$ genomic DNA, $0.5 \mu \mathrm{M}$ each primer, $200 \mu \mathrm{M}$ dNTPs, $1 \times$ reaction buffer, and $0.5 \mathrm{U}$ Taq DNA polymerase (Takara Bio Inc., Beijing, China). PCR amplification was performed on a PCR thermocycle instrument (Applied Biosystems, Foster, USA) using the following PCR programme: $94{ }^{\circ} \mathrm{C}$ for $5 \mathrm{~min}$; 35 cycles of $94{ }^{\circ} \mathrm{C}$ for $30 \mathrm{~s}, 50-60{ }^{\circ} \mathrm{C}$ for $30 \mathrm{~s}, 72^{\circ} \mathrm{C}$ for $30 \mathrm{~s}$; and a final $72{ }^{\circ} \mathrm{C}$ for $5 \mathrm{~min}$. Products were separated on the $8 \%$ polyacrylamide gel by electrophoresis. After electrophoresis at $220 \mathrm{~V}$ for $1.5 \mathrm{~h}$, the gel was separated from the plates and stained in $0.2 \% \mathrm{AgNO}_{3}$ solution (Shanghai Shi Yi chemicals Reagent, Shanghai, China). Finally, the stained gel was transferred into the developing solution (1.5\% sodium hydroxide and $0.4 \%$ formaldehyde) to reveal the silver-stained DNA bands. For SNP markers, the PCR reaction and PCR amplification were the same as for the SSR markers. While the reaction samples were $20 \mu \mathrm{l}$ in volume and the products were analysed by sequencing (Sangon Biotech, Shanghai, China).

\section{Map-based cloning}

The bulked segregation analysis (BSA) method [41] was performed in the $F_{2}$ population to screen for the linkage 
relationship between markers and the $u p$ locus. WT and mutant pools ( $\mathrm{M}$ pool) were constructed by mixing equal amounts of DNA from 10 downward-pedicel and 10 upward-pedicel plants, respectively. Polymorphic SSR markers and InDel markers between C-8-6 and up were identified and applied to analyse the WT and M pools. The up locus was mapped by using 70 individuals randomly selected from the small $F_{2}$ population (133 individuals). $933 \quad F_{2}$ individuals (from the small $F_{2}$ population with 133 individuals and the large $F_{2}$ population with 800 individuals) and $524 \mathrm{BC}_{1}$ individuals were used for fine mapping. SSR, InDel and SNP markers, which were used for fine mapping, are listed in Additional file 6: Table S1.

\section{Candidate gene prediction and gene annotation}

Candidate gene prediction was performed using the genome sequence of 'Gy14' in Phytozome v10 [42], the genome sequence of '9930' (http://cucurbitgenomics.org/ organism/2) [43] and the online program FGENESH (http://www.softberry.com/berry.phtml?topic=fgenesh\& group=programs\&subgroup=gfind. phtml) [44]. After gene annotation, the corresponding DNA fragments of candidate genes in this region were amplified from $u p$ and WT using KOD PLUS DNA polymerase (Toyobo, Osaka, Japan) for further sequencing.

\section{Gene expression analysis by real-time quantitative PCR (qRT-PCR)}

We used qRT-PCR to examine the pattern expression of Csa1G535800 in WT and up. Total RNA was extracted from roots, stems, true leaves, female flowers, male flowers, fruits and pedicels from opening female flowers. Reverse transcription was performed by $5 \times$ All-In-One RT MasterMix (ABM, Canada). The expression of Csa1G535800 in pedicels during different periods was also identified. Pedicels from 1 to $1.5 \mathrm{~cm}$ length fruits, the young fruits of the day before female flower open and the young fruits with opening female flowers were collected from WT and up. In order to exclude Csa1G535790 and Csa1G535810, we also detected their expression in the pedicels during different periods. The Total RNA was extracted by using the method mentioned above. Both the pattern expression and the temporal expression of Csa1G535800 were identified by using the primer pairs RT-1, which amplify the first exon. The expression of the gene Csa1G535800 was calculated by the comparative Ct method [45] based on the relative expression of the target gene versus the reference gene CsActin. Each test was repeated three times biologically and technically. The primer sequences for qRT-PCR are provided in Additional file 6: Table S1.

\section{Phylogenetic analysis}

Phylogenetic tree was constructed with the homology sequences of CsUP that were downloaded from the NCBI database including the following 10 species: Arabidopsis thaliana (Arabidopsis, accession no. NP_174319.1), Cucumis melo (melon, accession no. XP_008444679.1), Actinidia chinensis var. chinensis (Actinidia chinensis, accession no. PSS28916.1), Glycine max (soybean, accession no. XP_003535035.3), Jatropha curcas (Jatropha, accession no. XP_020535020.1), Nicotiana tabacum (tobacco, accession no. XP_016435663.1), Manihot esculenta (cassava, accession no. XP_021595454.1), Solanum lycopersicum (tomato, accession no. XP_004242447.1), Oryza sativa Japonica Group (rice, XP_015617894.1), Zea mays (maize, NP_001145320.1) and Gossypium hirsutum (cotton, accession no. XP_016688273.1). Protein sequence alignment was accomplished with ClustalW, and the neighbour-joining tree [46] was constructed with the MEGA 7.0 software package (http://www.megasoftware.net/) with 1000 bootstrap replications.

\section{Additional files} Additional file 1: Figure S1. Seven polymorphic markers between WT
and the M DNA pool (PDF $71 \mathrm{~kb}$ )

Additional file 2: Figure S2. Partial results of linkage analysis with indel-CsUp demonstrating that this marker is co-segregated with the upward-pedicel phenotype (PDF 91 kb)

Additional file 3: Figure S3. Pedicel orientation of B1 and CGN19839 indicating that both inbred lines have the upward-pedicel phenotype (PDF $271 \mathrm{~kb}$ )

Additional file 4: Figure S4. Structure of Csa1G535800 and two mutation sites in CGN19839. a Structure of Csa1G535800. Boxes and lines indicate exons and introns, respectively. Two green boxes in

Csa1G535800 indicate the 5' UTR and the 3' UTR. Sequencing results revealed that, compared to WT, CGN19839 has a SNP in the first exon and a 4-bp deletion in the sixth exon of Csa1G535800. The yellow box shows the deletion sequence in CGN19839. b The position of the SNP and the 4-bp deletion in the encoding sequence. Sequencing results revealed that there was a SNP ('A' in C-8-6 and ' $G$ ' in CGN19839) at $139 \mathrm{bp}$ and a 4-bp deletion at 1234-1237 bp from the ATG start codon (PDF 188 $\mathrm{kb})$

Additional file 5: Figure S5. Alignment of predicted protein sequences between WT and CGN19839. The amino acid highlighted in red is the acid alternation (Ile ${ }^{47}$ in WT to Val ${ }^{47}$ in CGN19839) caused by the SNP in the first exon of Csa1G535800. The amino acids highlighted in yellow are abnormal in CGN19839 and the amino acids highlighted in blue are missing in CGN19839. The DnaJ domain at the C-terminal is underlined (PDF $81 \mathrm{~kb}$ )

Additional file 6: Table S1. Primers used for mapping, cloning and qPCR. (PDF $186 \mathrm{~kb}$ ) (PDF $185 \mathrm{~kb}$ )

Additional file 7: Figure S6. Genomic DNA sequence alignment of CsUP from WT, up and CGN19839 (PDF $231 \mathrm{~kb}$ )

Additional file 8: Figure S7. Alignment of coding sequence of CSUP from WT and up with 19 other cucumber lines. The whole coding sequence length is $1404 \mathrm{bp}$. The $491-495 \mathrm{bp}$ position of up and B1 is the 5-bp deletion highlighted in blue. The 139 bp position of CGN19839 that altered from $A$ to $G$ is highlighted in red, and the $1234-1237$ bp position in CGN19839 is the 4-bp deletion in blue (PDF $451 \mathrm{~kb}$ ) 
Additional file 9: Figure S8. Genomic DNA sequence alignment of Cs535790 from WT and up (PDF $67 \mathrm{~kb}$ )

Additional file 10: Figure S9. Genomic DNA sequence alignment of Cs535810 from WT and up (PDF 43 kb)

Additional file 11: Figure S10. Alignment of promoter sequence of CsUP from WT and up. (PDF $108 \mathrm{~kb}$ )

Additional file 12: Figure S11. Alignment of promoter sequence of Csa1G535790 from WT and up. (PDF $109 \mathrm{~kb}$ )

Additional file 13: Figure S12. Alignment of promoter sequence of Csa1G535810 from WT and up. (PDF $108 \mathrm{~kb}$ )

Additional file 14: Figure S13. Expression level of Csa1G535790 and Csa1G535810 in pedicel at different stages. P1 pedicels from 1 to $1.5 \mathrm{~cm}$ length young fruits; $\mathrm{P} 2$ pedicels from the young fruits of the day before female flowers open; P3 pedicels from the young fruits with opening female flowers. Data are displayed as the ratio of expression to CsActin3 with three biological replicates. Error bars represent standard error (SE) (PDF $133 \mathrm{~kb}$ )

\section{Abbreviations}

BSA: Bulked segregation analysis; DAP: Day after pollination; InDel: Insertiondeletion; SNP: Single nucleotide polymorphism; SSR: Simple Sequence Repeats; WT: Wild type

\section{Acknowledgements}

We would like to thank Dr. Yi-qun Weng (Horticulture Department, University of Wisconsin Madison, USA) for affording information about the cucumber genetic map.

\section{Consent to publication}

Not applicable.

\section{Funding}

This study was supported by The National Key R\&D Program of China (Grant No. 2018YFD0100701), the Science and Technology Commission of Shanghai Municipality (No: 18391900300), the National Natural Science Foundation of China (No. 31672148), the Youth Fund of the Natural Science and Agri-X Project of Shanghai Jiao Tong University (AgriX2015002), and the Shanghai Graduate Education and Innovation Programme (Horticulture).

\section{Availability of data and materials}

All data generated or analyzed during this study are included in this published article [and its additional files].

\section{Authors' contributions}

JS performed the experiments and prepared the manuscript. TX contributed to the data analysis. JN founded our experiments. YC, DL, MP and QG contributed to the extraction of DNA. CG and LZ contributed to the writing of this paper. $\mathrm{HH}$ contributed to taking care of the plants. $\mathrm{HL}$, JP and RC offered good advice about the experiments. GW, the corresponding authors, designed the experiments, supervised this study and wrote the manuscript. All authors have read and approved the manuscript.

\section{Ethics approval and consent to participate}

Not applicable.

\section{Competing interests}

The authors declare that they have no competing financial interests.

\section{Publisher's Note}

Springer Nature remains neutral with regard to jurisdictional claims in published maps and institutional affiliations.

Received: 26 November 2018 Accepted: 11 April 2019 Published online: 25 April 2019

\section{References}

1. Bisognin DA. Origin and evolution of cultivated cucurbits Origem e evolução de cucurbitáceas cultivadas. Ciênc Rural. 2002;32(4):715-23.
2. Li Y, Wen C, Weng Y. Fine mapping of the pleiotropic locus B for black spine and orange mature fruit color in cucumber identifies a $50 \mathrm{~kb}$ region containing a R2R3-MYB transcription factor. Theor Appl Genet. 2013;126(8): 2187-96.

3. Sebastian P, Schaefer H, Telford IRH, Renner SS. Cucumber (Cucumis sativus) and melon (C. melo) have numerous wild relatives in Asia and Australia, and the sister species of melon is from Australia. Proc Natl Acad Sci. 2010; 107(32):14269-73.

4. Yundaeng C, Somta P, Tangphatsornruang S, Chankaew S, Srinives P. A single base substitution in BADH/AMADH is responsible for fragrance in cucumber (Cucumis sativus L.), and development of SNAP markers for the fragrance. Theor Appl Genet. 2015;128(9):1-12.

5. Zhao J, Li Y, Ding L, Yan S, Liu M, Jiang L, Zhao W, Wang Q, Yan L, Liu R. Phloem transcriptome signatures underpin the physiological differentiation of the pedicel, stalk and fruit of cucumber (Cucumis sativus L.). Plant Cell Physiol. 2015;57(1):19-34.

6. Eisikowitch D, Rotem R. Flower orientation and color change in Quisqualis indica and their possible role in pollinator partitioning. Bot Gaz. 1987;148(2): 175-9.

7. Kevan PG. Sun-tracking solar furnaces in high arctic flowers: significance for pollination and insects. Science. 1975;189(4204):723-6.

8. Huang SQ, Takahashi Y, Dafni A. Why does the flower stalk of Pulsatilla cernua (Ranunculaceae) bend during anthesis? Am J Bot. 2002;89(10):1599_ 603.

9. Patiño S, Jeffree C, Grace J. The ecological role of orientation in tropical convolvulaceous flowers. Oecologia. 2002;130(3):373-9.

10. Ushimaru A, Hyodo F. Why do bilaterally symmetrical flowers orient vertically? Flower orientation influences pollinator landing behaviour. Evol Ecol Res. 2005;7(9):151-60.

11. Ushimaru A, Dohzono I, Takami Y, Hyodo F. Flower orientation enhances pollen transfer in bilaterally symmetrical flowers. Oecologia. 2009;160(4):667.

12. Tadey M, Aizen MA. Why do flowers of a hummingbird-pollinated mistletoe face down? Funct Ecol. 2001;15(6):782-90

13. Ushimaru A, Kawase D, Imamura A. Flowers adaptively face down-slope in 10 forest-floor herbs. Funct Ecol. 2006;20(4):585-91.

14. Wang H, Tie S, Yu D, Guo YH. Yang CF. change of floral orientation within an inflorescence affects pollinator behavior and pollination efficiency in a bee-pollinated plant, Corydalis sheareri. PLoS One. 2014;9(4):e95381.

15. Komeda Y, Takahashi T, Hanzawa Y. Development of inflorescences in Arabidopsis thaliana. J Plant Res. 1998;111(2):283-8.

16. Venglat SP, Dumonceaux T, Rozwadowski K, Parnell L, Babic V, Keller W, Martienssen R, Selvaraj G, Datla R. The homeobox gene BREVIPEDICELLUS is a key regulator of inflorescence architecture in Arabidopsis. Proc Natl Acad Sci. 2002;99(7):4730-5.

17. $X u L, X u Y$, Dong A, Sun Y, Pi L, Xu Y, Huang H. Novel as1 and as 2 defects in leaf adaxial-abaxial polarity reveal the requirement for ASYMMETRIC LEAVES1 and 2 and ERECTA functions in specifying leaf adaxial identity. Development. 2003;130(17):4097-107

18. Douglas SJ, Riggs CD. Pedicel development in Arabidopsis thaliana : contribution of vascular positioning and the role of the BREVIPEDICELLUS and ERECTA genes. Dev Biol. 2005;284(2):451-63.

19. Yamaguchi N, Suzuki M, Fukaki H, Morita-Terao M, Tasaka M, Komeda Y. CRM1/BIG-mediated auxin action regulates Arabidopsis inflorescence development. Plant Cell Physiol. 2007;48(9):1275-90.

20. Ragni L, Bellesboix E, Günl M, Pautot $V$. Interaction of KNAT6 and KNAT2 with BREVIPEDICELLUS and PENNYWISE in Arabidopsis inflorescences. Plant Cell. 2008;20(4):888-900.

21. Douglas SJ, Chuck G, Dengler RE, Pelecanda L, Riggs CD. KNAT1 and ERECTA regulate inflorescence architecture in Arabidopsis. Plant Cell. 2002 14(3):547.

22. Guo M, Thomas J, Collins G, Timmermans MC. Direct repression of KNOX loci by the ASYMMETRIC LEAVES1 complex of Arabidopsis. Plant Cell. 2008; 20(1):48-58.

23. Li Y, Pi L, Huang H, Xu L. ATH1 and KNAT2 proteins act together in regulation of plant inflorescence architecture. J Exp Bot. 2011;63(3):1423-33.

24. Ori N, Eshed Y, Chuck G, Bowman JL, Hake S. Mechanisms that control knox gene expression in the Arabidopsis shoot. Development. 2000;127(24):5523-32

25. Semiarti E, Ueno Y, Tsukaya H, Iwakawa H, Machida C, Machida Y. The ASYMMETRIC LEAVES2 gene of Arabidopsis thaliana regulates formation of a symmetric lamina, establishment of venation and repression of meristemrelated homeobox genes in LEAVES. Development. 2001;128(10):1771-83. 
26. Smith HM, Campbell BC, Hake S. Competence to respond to floral inductive signals requires the homeobox genes PENNYWISE and POUND-FOOLISH. Curr Biol. 2004;14(9):812-7.

27. Yamaguchi N, Yamaguchi A, Abe M, Wagner D, Komeda Y. LEAFY controls Arabidopsis pedicel length and orientation by affecting adaxial-abaxial cell fate. Plant J. 2012;69(5):844-56

28. Wang D, Chen X, Zhang Z, Liu D, Song G, Kong X, Geng S, Yang J, Wang B, Wu L. A MADS-box gene NtSVP regulates pedicel elongation by directly suppressing a KNAT1-like KNOX gene NtBPL in tobacco (Nicotiana tabacum L.). J Exp Bot. 2015;66(20):6233-44.

29. Lin D, Xiang Y, Xian Z, Li Z. Ectopic expression of SIAGO7 alters leaf pattern and inflorescence architecture and increases fruit yield in tomato. Physiol Plant. 2016;157(4):490-506.

30. Cheng J, Qin C, Tang X, Zhou H, Hu Y, Zhao Z, Cui J, Li B, Wu Z, Yu J. Development of a SNP array and its application to genetic mapping and diversity assessment in pepper (Capsicumspp.). Sci Rep. 2016;6:33293.

31. Lin WC, Shuai B, Springer PS. The Arabidopsis LATERAL ORGAN BOUNDARIES-domain gene ASYMMETRIC LEAVES2 functions in the repression of KNOX gene expression and in adaxial-abaxial patterning. Plant Cell. 2003;15(10):2241.

32. Dong Y, Liu J, Li P-W, Li C-Q, Lü T-F, Yang X, Wang Y-Z. Evolution of Darwin's peloric gloxinia (Sinningia speciosa) is caused by a null mutation in a pleiotropic TCP gene. Mol Biol Evol. 2018;35(8):1901-15.

33. Adamowski M, Narasimhan M, Kania U, Glanc M, De Jaeger G, Friml J. A functional study of AUXILIN-LIKE1 and 2, two putative Clathrin Uncoating factors in Arabidopsis. Plant Cell. 2018;30(3):700-16.

34. Suetsugu N, Kagawa T, Wada M. An auxilin-like J-domain protein, JAC1, regulates phototropin-mediated chloroplast movement in Arabidopsis. Plant Physiol. 2005;139(1):151-62

35. Park C-J, Wei T, Sharma R, Ronald PC. Overexpression of rice auxilin-like protein, XB21, induces necrotic lesions, up-regulates endocytosis-related genes, and confers enhanced resistance to Xanthomonas oryzae pv. oryzae. Rice. 2017;10(1):27.

36. Yang KZ, Xia C, Liu XL, Dou XY, Wang W, Chen LQ, Zhang XQ, Xie LF, He L, Ma X. A mutation in Thermosensitive Male Sterile 1, encoding a heat shock protein with DnaJ and PDI domains, leads to thermosensitive gametophytic male sterility in Arabidopsis. Plant J. 2009:57(5):870-82.

37. Ezaki B, Kiyohara H, Matsumoto H, Nakashima S. Overexpression of an auxilin-like gene (F9E10. 5) can suppress Al uptake in roots of Arabidopsis. J Exp Bot. 2006;58(3):497-506.

38. Kampinga HH, Craig EA. The HSP70 chaperone machinery: J proteins as drivers of functional specificity. Nat Rev Mol Cell Biol. 2010;11(8):579.

39. Lemmon SK. Clathrin uncoating: Auxilin comes to life. Curr Biol. 2001;11(2): R49-52.

40. Murray MG, Thompson WF. Rapid isolation of high molecular weight plant DNA. Nucleic Acids Res. 1980:8(19):4321-5.

41. Michelmore RW, Paran I, Kesseli R. Identification of markers linked to disease-resistance genes by bulked segregant analysis: a rapid method to detect markers in specific genomic regions by using segregating populations. Proc Natl Acad Sci. 1991:88(21):9828-32.

42. Cavagnaro PF, Senalik DA, Yang L, Simon PW, Harkins TT, Kodira CD, Huang $\mathrm{S}$, Weng Y. Genome-wide characterization of simple sequence repeats in cucumber (Cucumis sativus L.). BMC Genomics. 2010;11(1):1-18.

43. Huang S, Li R, Zhang Z, Li L, Gu X, Fan W, Lucas WJ, Wang X, Xie B, Ni P. The genome of the cucumber, Cucumis sativus L. Nat Genet. 2009;41(12): 1275

44. Salamov AA, Solovyev W. Ab initio gene finding in Drosophila genomic DNA. Genome Res. 2000;10(4):516

45. Simon P. Q-Gene: processing quantitative real-time RT-PCR data. Bioinformatics. 2003;19(11):1439-40.

46. Saitou N, Nei M. The neighbor-joining method: a new method for reconstructing phylogenetic trees. Mol Biol Evol. 1987;4(4):406-25.

Ready to submit your research? Choose BMC and benefit from:

- fast, convenient online submission

- thorough peer review by experienced researchers in your field

- rapid publication on acceptance

- support for research data, including large and complex data types

- gold Open Access which fosters wider collaboration and increased citations

- maximum visibility for your research: over $100 \mathrm{M}$ website views per year

At BMC, research is always in progress.

Learn more biomedcentral.com/submissions 